\title{
LINC00470 accelerates the proliferation and metastasis of melanoma through promoting APEX1 expression
}

\author{
Ting Huang ${ }^{1}$, Yong-Jie Wang ${ }^{1}$, Mi-Tao Huang ${ }^{2}$, Yu Guo ${ }^{1}$, Li-Chang Yang ${ }^{1}$, Xiao-Jin Liu', Wu-Yuan Tan ${ }^{1}$ and \\ Jian-Hong Long (1)
}

\begin{abstract}
Recently studies found that APEX1 was abnormally expressed in melanoma, indicating that it might be involved in the development of melanoma. However, the underlying mechanism and the interaction between APEX1 and LINC00470 in melanoma are not clear. Therefore, we aimed to investigate the role of LINC00470 in the development of melanoma in this work. We discovered that LINC00470 was overexpressed in melanoma tissues and cells compared with the adjacent normal tissues and cells by qPCR. The overexpression of LINC00470 promoted the proliferation and migration of melanoma cells. The functional investigation demonstrated that LINC00470 activated the transcription factor, ZNF131, to regulate the APEX1 expression, which finally promoted cell proliferation and migration. In contrast, knockdown of LINC00470 could significantly inhibit the melanoma cell proliferation and migration, and suppress the growth of tumor in vivo. Overexpression of APEX1 could reverse the impact of the silence of LINC00470 in melanoma cells. In summary, our studies revealed that LINC00470 promoted melanoma proliferation and migration by enhancing the expression of APEX1, which indicated that LINC00470 might be a therapeutic target for the treatment of melanoma.
\end{abstract}

\section{Introduction}

Melanoma is recognized as the most aggressive skin cancer with rapid progression, stemming from melanocytes $^{1,2}$. Every year, the incidence of melanoma is rapidly increased $^{3}$. Around the world, melanoma ranks as the seventh most common cancer for females and the fifth most common cancer in male ${ }^{4}$. Melanoma is also characterized by the most common cancer with metastasis. Patients are usually diagnosed with melanoma at a late stage with metastasis, resulting in a poor prognosis. The 5 -year survival rate of melanoma was reported to be less than $18 \%^{5,6}$. Therefore, it is urgent and important to

Correspondence: Jian-Hong Long (jh_plastic@163.com)

'Department of Plastic Surgery, Xiangya Hospital, Central South University, Changsha 410011 Hunan Province, P. R. China

2Department of Burns and Reconstructive Surgery, Xiangya Hospital, Central South University, Changsha 410011 Hunan Province, P. R. China

Edited by E. Candi explorer the molecular mechanism of the development of melanoma, which might provide potential therapeutic targets for melanoma treatment.

Apurinic/apyrimidinic endonuclease 1/redox effector factor 1 (APEX1), an important multifunctional protein, not only works as a transcriptional coactivator in cell apoptosis, proliferation, and differentiation but also regulates DNA repair and adjustment of intracellular reactive oxygen species $^{7,8}$. Therefore, APEX1 has been studied and worked as a biomarker for a variety of cancers. For example, Cao et al. ${ }^{9}$ found that APEX1 was overexpressed in the hepatocellular carcinoma (HCC) tissues and cells, which indicated that APEX1 could be used for the diagnosis and prognosis of HCC. APEX1 was also upregulated in non-small-cell lung cancer and co-related with the high cell proliferation and metastasis ${ }^{10}$. Besides these two types of cancer, abnormal expression of APEX1 in both mRNA and protein levels have been found in other cancers, such

\section{(c) The Author(s) 2021}

(c) Open Access This article is licensed under a Creative Commons Attribution 4.0 International License, which permits use, sharing, adaptation, distribution and reproduction cc) in any medium or format, as long as you give appropriate credit to the original author(s) and the source, provide a link to the Creative Commons license, and indicate if changes were made. The images or other third party material in this article are included in the article's Creative Commons license, unless indicated otherwise in a credit line to the material. If material is not included in the article's Creative Commons license and your intended use is not permitted by statutory regulation or exceeds the permitted use, you will need to obtain permission directly from the copyright holder. To view a copy of this license, visit http://creativecommons.org/licenses/by/4.0/. 
as breast cancer ${ }^{11}$, gastric cancer ${ }^{12}$, ovarian serous cancer $^{13}$, bladder cancer ${ }^{14}$, colon cancer ${ }^{15}$, prostate cancer ${ }^{16}$, osteosarcoma cancer ${ }^{17}$, melanoma ${ }^{18}$, etc. Overexpression (OE) of APEX1 in melanoma was clearly observed by both Yang et al. ${ }^{18}$ as well as Figi et al. ${ }^{19}$, showing that APEX1 might involve in the development of melanoma. However, the regulation of APEX1 in melanoma and its mechanism is unclear, which limited the discovery of its related potential therapeutic strategies.

Long non-coding RNAs (LncRNAs) with no proteincoding ability, are a new type of RNAs with more than 200 nucleotides. LncRNAs act as functional regulators in a variety of cancers ${ }^{20,21}$. LncRNAs regulate target gene expression and conduct their function in cancer through various mechanisms, such as chromatin modification, RNA editing/splicing/degradation, miRNA sequestration, transcriptional activation/repression, and translational efficiency modulation ${ }^{22}$. In melanoma, LncRNAs also play important roles in cell proliferation, invasion, and migration. For example, Chen et al. ${ }^{23}$ found that LncRNA GAS5 could inhibit tumor growth through regulating the metastasis of melanoma cells. Zhang et al. ${ }^{6}$ discovered that LncRNA HOXD-AS1 promoted melanoma cell proliferation and invasion by inhibiting the expression of RUNX3. LINC00470, identified as C18orf2, is a LncRNA located in chromosome band $18 \mathrm{p} 11.32^{24,25}$. Recently, LINC00470 was found highly expressed in the glioblastoma tissues and cells, which activated AKT signaling and promoted the expression of ELFN2 to inhibit cell autophagy and induce tumorigenesis ${ }^{26,27}$. Yan et al. found that LINC00470 was significantly upregulated in gastric cancer cells compared with normal tissues and cells. Meanwhile, they demonstrated that LINC00470 induced the degradation of PTEM mRNA to promote gastric cancer proliferation, migration, and invasion ${ }^{28}$. However, the functions and molecular mechanisms of LINC00470 remain unclear in melanoma.

In the study, we found that LINC00470 and APEX1 were significantly overexpressed in the tissues and cells of melanoma. Furthermore, upregulation of LINC00470 significantly promoted cell proliferation and migration. Knockdown of LINC00470 in vivo reduced tumor growth. In addition, we discovered that LINC00470 activated the transcription factor ZNF131 to promote the OE of APEX1 in protein level, but not in mRNA level. Therefore, we believe that LINC00470 may be used as a potential therapeutic target of melanoma.

\section{Materials and methods}

\section{Tissue specimens and cell lines}

Melanoma cancer tissues and healthy tissues from 20 patients were obtained during tumor removal surgery with their signed statement of informed consent. Those tissues were kept at $-80^{\circ} \mathrm{C}$ in liquid nitrogen before usage. Approvements were made by the ethics committee of Central South University.

The human melanoma cell lines, including A375, A875, SK-MEL-1, SK-MEL-5, and SK-MEL-28, as well as normal human epidermal melanocytes (PIG1), were obtained from the American Type Culture Collection (ATCC, Manassas, VA). RPMI-1640 or DMEM or 254 medium supplemented with $10 \%$ fetal bovine serum (FBS) were used to culture cells at $37^{\circ} \mathrm{C}$ in a humidified chamber with $5 \% \mathrm{CO}_{2}$.

\section{Cell transfection}

All the short hairpin (sh) RNAs, including shNC, shAPEX1, shLINC00470, and shZNF131 were synthesized by GenePharma (Shanghai, China). The following shows all the sequences: shNC: $5^{\prime}$-UCUAUGUGUCUUAAUC CCUUGUCCU-3'; sh-APEX1: 5'-GACACGCGACUUGU ACCAC-3'; shLINC00470: 5'-GACACGCGACUUGUAC CAC-3'; shZNF131: 5'-GACACGCGACUUGUACCAC$3^{\prime}$. In addition, pcDNA3.1 empty vector (Invitrogen, Shanghai, China) was inserted with the cDNA sequences of AR, APEX1, LINC00470, and ZNF131 to construct the OE vectors, including OE-AR, OE-APEX1, OELINC00470, and OE-ZNF131, respectively. Lipofectamine 3000 transfection reagent (Invitrogen) was used to transfect cells following the manufacturer's instructions.

\section{Quantitative real-time polymerase chain reaction (qRT- PCR)}

TRIzol reagent (Invitrogen, Carlsbad, CA) was used to collect total RNA. Then, the PrimeScript ${ }^{\mathrm{TM}} \mathrm{RT}$ Reagent kit (Takara Bio. Inc) was utilized to obtain the cDNA through reverse transcription. All the primers were obtained from GenePharma (Shanghai, China). The DNA primers sequences: APEX1 forward, $5^{\prime}$-tgggcttccagagcttt cta- $3^{\prime}$ and reverse, $5^{\prime}$-ttctttattgagggcaaccg-3'; LINC00470 forward, $5^{\prime}$-agggacaatctcacacagg- $3^{\prime}$ and reverse, $5^{\prime}$-gact caaccttcctctcca-3'; ZNF131 forward, $5^{\prime}$-agggacaatctcacac agg- $3^{\prime}$ and reverse, $5^{\prime}$-gactcaaccttcctctcca- $3^{\prime}$; GAPDH forward, $5^{\prime}$-gtttgtgatgggtgtgaacc- $3^{\prime}$ and reverse, $5^{\prime}$-tcttct gagtggcagtgatg- $3^{\prime}$. mRNA levels were determined by qRTPCR on an ABI Prism 7900HT (Applied Biosystems, Foster City, CA USA). The $\Delta \Delta$ Cq method was used to quantify the Cq-value for every sample, whose results were expressed as $2^{-\Delta \Delta \mathrm{Cq}}$. Each sample was performed in triplicate.

\section{LncRNA sequencing}

LncRNA sequencing was applied to analyze the expression of LncRNAs in the AR-overexpressed A375 cells and A375 cells. First, total RNAs were collected by TRIzol reagent (Invitrogen). Ribosomal RNA (rRNA) was discarded using Epicenter Ribo-Zero ${ }^{\mathrm{TM}}$ rRNA Removal Kit (Epicenter, USA) from the total RNA. Then, cDNA 
libraries were obtained with the Illumina TruSeq Stranded Total RNA using RiboZero Human Kit (Illumina, San Diego, CA). Finally, the sequencing was performed by Illumina HiSeq. 2000 platform. The RNA-seq dataset was analyzed and demonstrated by the Integrative Genomics Viewer.

\section{RNA immunoprecipitation (RIP) assay}

RIP assay was utilized to investigate the relationship between LINC00470 and transcription factor ZNF131 in melanoma tissues and cells. An EZ-Magna RIP ${ }^{\mathrm{TM}}$ RNABinding Protein Immunoprecipitation Kit (Millipore, USA) and anti-ZNF131 antibody (Invitrogen, PA5-43065 were utilized in the RIP assay following the manufacturer's instructions. Melanoma tissues and cells were lysed by the buffer provided in the kit. Then, anti-ZNF131 or control IgG (negative control) was applied for immunoprecipitation. qRT-PCR was conducted to quantify the LINC00470 level after purification with RNAiso Plus (Takara, Japan).

\section{Western blot analysis}

Briefly, the cells or tissues were lysed using RIPA lysis buffer (Beijing Solarbio Science \& Technology Co., Ltd., Beijing, China). The total proteins were quantified with a BCA assay kit (Pierce; Fisher Scientific, Inc.). The antibodies were used as follows: anti-APEX1 (1:10,000, Proteintech, 10203-1-AP, anti-ZNF131 (1:10,000, Invitrogen, PA5-43065), anti-AR (1:10,000, Abcam) and anti-GAPDH (1:10,000; cat. no. ab181602; Abcam). At room temperature, the membranes were incubated with all the primary antibodies for $2 \mathrm{~h}$. Then, PBS was used to wash the membrane three times, followed by incubating with secondary antibodies for another $1 \mathrm{~h}$ at room temperature. The signals were obtained through the Chemiluminescence Western blot system (Pierce, Biotechnology Inc., Rockford, USA). Each sample repeated at least three times.

\section{MTT assay}

Cell proliferation was assessed by MTT assay using MTT reagent (Roche Molecular Biochemicals, Rotkreuz, Switzerland). Totally, 2000 cells were seeded in 96-well plates per well for different treatments. After different treatments for 24,48 , and $72 \mathrm{~h}$, the MTT reagent was pipetted into each well and incubated for another $4 \mathrm{~h}$. After removing the medium, $200 \mu \mathrm{L}$ DMSO was injected into each well. The absorbance at $490 \mathrm{~nm}$ was collected on a microplate reader.

\section{Colony formation assay}

The cells with a density of 5000 cells/well were seeded in a 6-well plate for different treatments for 15 days. Then, methanol was used to fix the cells, followed by the incubation of $0.1 \%$ crystal violet. The colonies were observed and quantified with the optical microscope. Each sample was repeated in triplicates.

\section{Transwell assay}

A Transwell assay was utilized to determine the migration ability of cells with a two-chamber with an $8 \mu \mathrm{m}$ pore size membrane filter (Corning, Cambridge, MA). Briefly, cells with different treatments were seeded in the top chamber in the serum-free medium. In the lower chamber, a complete medium containing $10 \%$ FBS was used. We incubated the chambers at $37^{\circ} \mathrm{C}$ for $48 \mathrm{~h}$. After removing the cells in the top chamber, the cells in the lower chamber were fixed with methanol, after which $0.1 \%$ crystal violet was used to stain the cells for $30 \mathrm{~min}$. Then, the stained cells were imaged and counted under the microscope. Three independent experiments were conducted for each sample.

\section{Luciferase reporter assay}

The luciferase reporter assay was conducted according to the previous literature ${ }^{27}$. The cells were co-transfected with psiCHECK2 plasmid (Promega, Madison, WI) containing the APEX1 promoter and ZNF131 wild-type (WT) or mutant for $48 \mathrm{~h}$. Dual-Glo Luciferase Assay System (Promega) was used to quantify the luciferase reporter activity following the manufacturer's protocol.

\section{Generation of xenografts}

The animal experiments were approved by the Animal Ethics Committee of Central South University. Briefly, xenografts were generated by injecting the A375 cells $(2 \times$ $10^{6}$ in $0.1 \mathrm{ml} \mathrm{PBS)}$ subcutaneously into six-week-old $\mathrm{BALB} / \mathrm{c}$ male athymic nude mice $(n=5 /$ group, Vitalriver, Beijing, China). Then, the tumor sizes were monitored for 28 days every 7 days (tumor volume $=\left(\right.$ length $\times$ width $\left.^{2}\right) /$ 2 ). On day 28 , the tumor tissues from each group were collected after the mice were sacrificed. The obtained tissues were used for further analysis.

\section{IHC analysis}

The resected tumor tissues from each group were fixed using $10 \%$ formalin and stored in paraffin for section. Then, the samples were stained with hematoxylin and eosin. The anti-ZNF131 antibody (diluted at 1:100, Invitrogen, PA5-43065), anti-Ki67 antibody (diluted at 1:500, Abcam, ab15580) and anti-APEX1 antibody (diluted at 1:100, Proteintech, 10203-1-AP) were utilized for IHC staining according to the manufacture's instruction.

\section{RNA pull-down}

RNA pull-down assay was also used to investigate the relationship between LINC00470 and transcription factor ZNF131 in melanoma cells. The biotin-coupled RNA 
complex was pulled down by incubating the melanoma cell lysates with streptavidin-coated magnetic beads (Sigma, USA) following the manufacturer's instructions. Real-time PCR analysis was used to evaluate the enrichment of LINC00470 in the capture fractions. LINC0047 junction probe, control probe is ordered from Sangon Biotech (Shanghai, China). The proteins in the capture complex were identified by western blotting using an antiZNF131 antibody.

\section{Chromatin Immunoprecipitation (CHIP) assay}

To study the relationship between transcription factor ZNF131 and APEX1 in melanoma cells, we performed a CHIP assay using a SimpleChIP ${ }^{\circledR}$ Enzymatic Chromatin IP Kit (CST, USA). Briefly, the cultured melanoma cells were lysed and sonicated to chromatins. The chromatins were incubated with anti-ZNF131 antibodies or normal rabbit IgG overnight. Real-time PCR analysis was used to quantify the APEX1 level in the enrichment of specific DNA sequences. The primers used for ChIP are ordered from Sangon Biotech (Shanghai, China).

\section{Statistical analysis}

Data are shown as the means \pm standard deviation (SD). Every group was repeated at least three times. Student's $t$ test or one-way ANOVA was utilized to compare the difference. $p<0.05$ was defined as statistically significant differences $\left(" p<0.05\right.$, ${ }^{* *} p<0.01$, and $\left.{ }^{* * *} p<0.001\right)$.

\section{Results}

\section{APEX1 promoted the melanoma cell proliferation and migration}

To investigate the function of APEX1 in melanoma, we transfected the OE or silence vector of APEX1 into the A375 and SK-MEL-5 cells. As shown in Fig. 1A, B, A375 and SK-MEL-5 cells transfected with OE-APEX1 showed significantly enhanced expression of APEX1 compared with the OE-NC group. In contrast, transfection with shAPEX1 significantly decreased the expression of APEX1 compared with shNC group. These results indicated that the OE or silence of APEX1 was successfully fulfilled in A375 and SK-MEL-5 cells. MTT and colony formation assay was used to assess the cell proliferation after overexpressing or silencing APEX1. The results demonstrated that cell proliferation was promoted when the APEX1 was overexpressed in A375 and SK-MEL-5 cells (Fig. 1C-F). In contrast, silencing APEX1 significantly suppressed cell proliferation (Fig. 1C-F). Furthermore, the impact of APEX1 on migration ability was investigated using transwell assay in the melanoma cells. We found that the OE of APEX1 significantly promoted the cell migration ability (Fig. 1G, H). Inversely, the knockdown of APEX1 suppressed cell migration of A375 and SK-MEL-5 cells (Fig. 1G, H). Overall, these results indicated that
APEX1 promoted the proliferation and migration ability of melanoma cells.

\section{Linc 00470 overexpressed in the melanoma tissues and cells}

Androgen receptor (AR) involves in the development of melanoma. To discover the function of the lncRNAs in the AR-induced melanoma development, we studied the expression of lncRNAs in AR-overexpressed melanoma cells using lncRNA sequencing analysis. Many abnormally expressed lncRNAs in the AR-overexpressed A375 cells were identified (Fig. 2A). LINC00662 and LINC00470 ranked as one of the top 60 remarkably upregulated expressed lncRNAs with relatively high abundance (Fig. 2B), which was validated by qRT-PCR analysis (Fig. 2C). Meanwhile, we detected LINC00470 expression in 20 paired melanoma tissue and adjacent normal tissue by qRT-PCR, and the expression of LINC00470 was significantly higher in melanoma tissues (Fig. 2D). In addition, LINC00470 expression was upregulated in three melanoma cells (A375, SK-MEL-1, and SK-MEL-5), compared with the human normal skin melanocytes cell line PIG1 (Fig. 2E). Taken together, these results demonstrated that the LINC00470 was upregulated in melanoma tissues and cells, which might be used as a melanoma prognostic marker.

\section{LINC00470 promoted the proliferation and migration of melanoma cells}

The role of LINC00470 on cell proliferation and migration ability was investigated by overexpressing or silencing LINC00470 in A375 and SK-MEL-5 cells. The qRT-PCR analysis demonstrated that LINC00470 was successfully silenced and overexpressed in A375 and SKMEL-5 cells, which were transfected with shLINC00470 and OE-LINC00470, respectively (Fig. 3A, B). MTT and colony-forming assay revealed that OE of LINC00470 could significantly promote cell proliferation. In contrast, knockdown of LINC00470 led to significant inhibition of cell proliferation (Fig. 3C-F). Moreover, overexpressing LINC00470 significantly increased melanoma cell migration (Fig. 3G, H). However, knocking down LINC00470 suppressed cell migration ability in A375 and SK-MEL-5 cells (Fig. 3G, H). Therefore, we concluded that LINC00470 promoted cell proliferation and migration in A375 and SK-MEL-5 cells.

\section{LINC00470 promoted melanoma cell proliferation and migration through increasing APEX1}

From the previous results, we found both LINC00470 and APEX1 were upregulated in melanoma cells. Subsequently, we investigated the detailed relationship between LINC00470 and APEX1 in melanoma cells. In the ARoverexpressed A375 cells, western blot analysis 


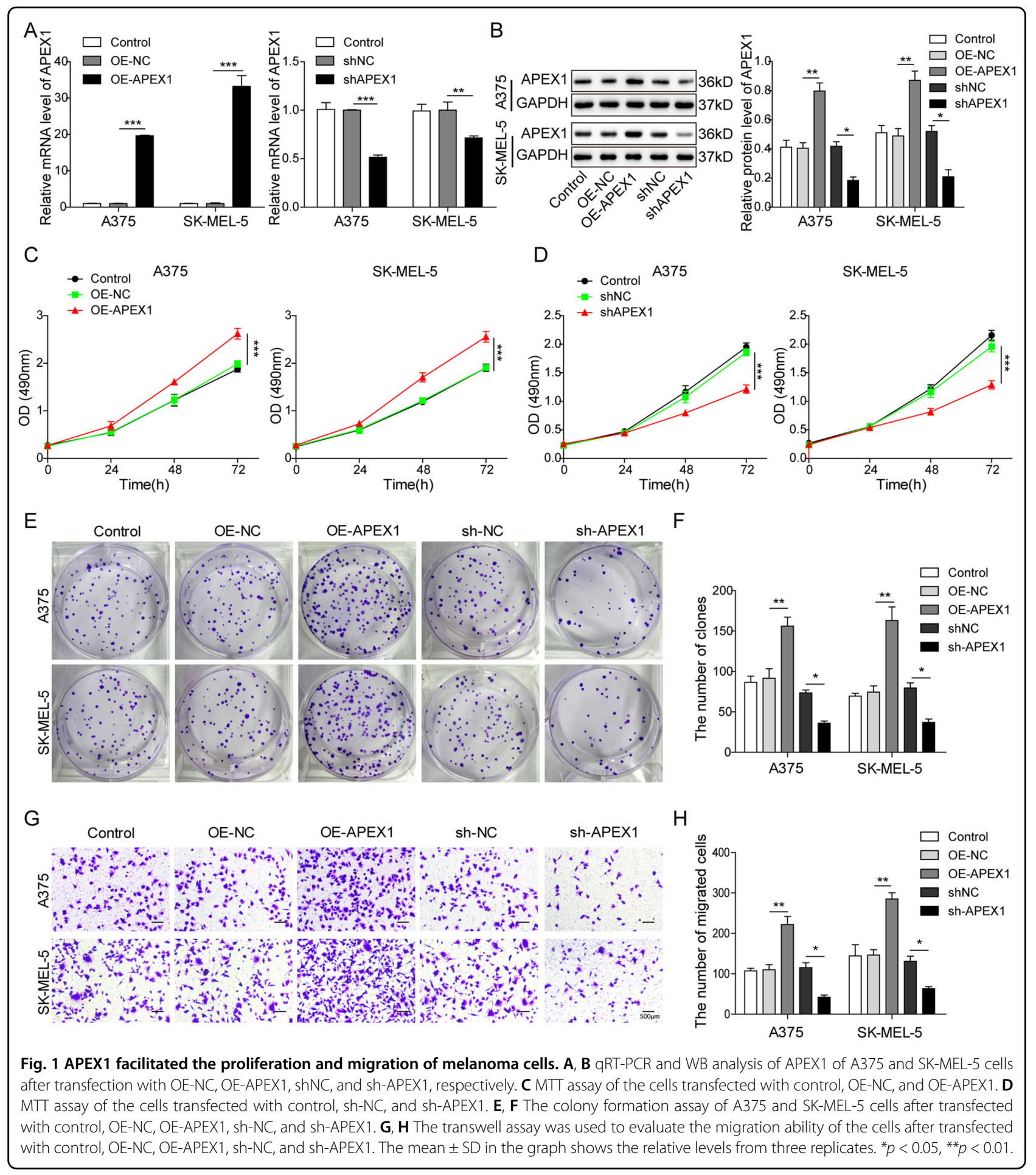

demonstrated that AR and APEX1 were both upregulated (Fig. S1). As shown in Fig. 4A, OE LINC00470 inhibited while knockdown LINC00470 promoted the mRNA expression level of APEX1 in melanoma cells. Interestingly, the protein expression level of APEX1 was increased when the melanoma cells were overexpressed LINC00470 and decreased when LINC00470 was knockdown (Fig. 4B). These results implied that LINC00470 might impact the protein expression level of APEX1 through the regulation of posttranscriptional translation. Then, MTT and colony formation assay demonstrated that silencing LINC00470 suppressed the cell proliferation ability but 


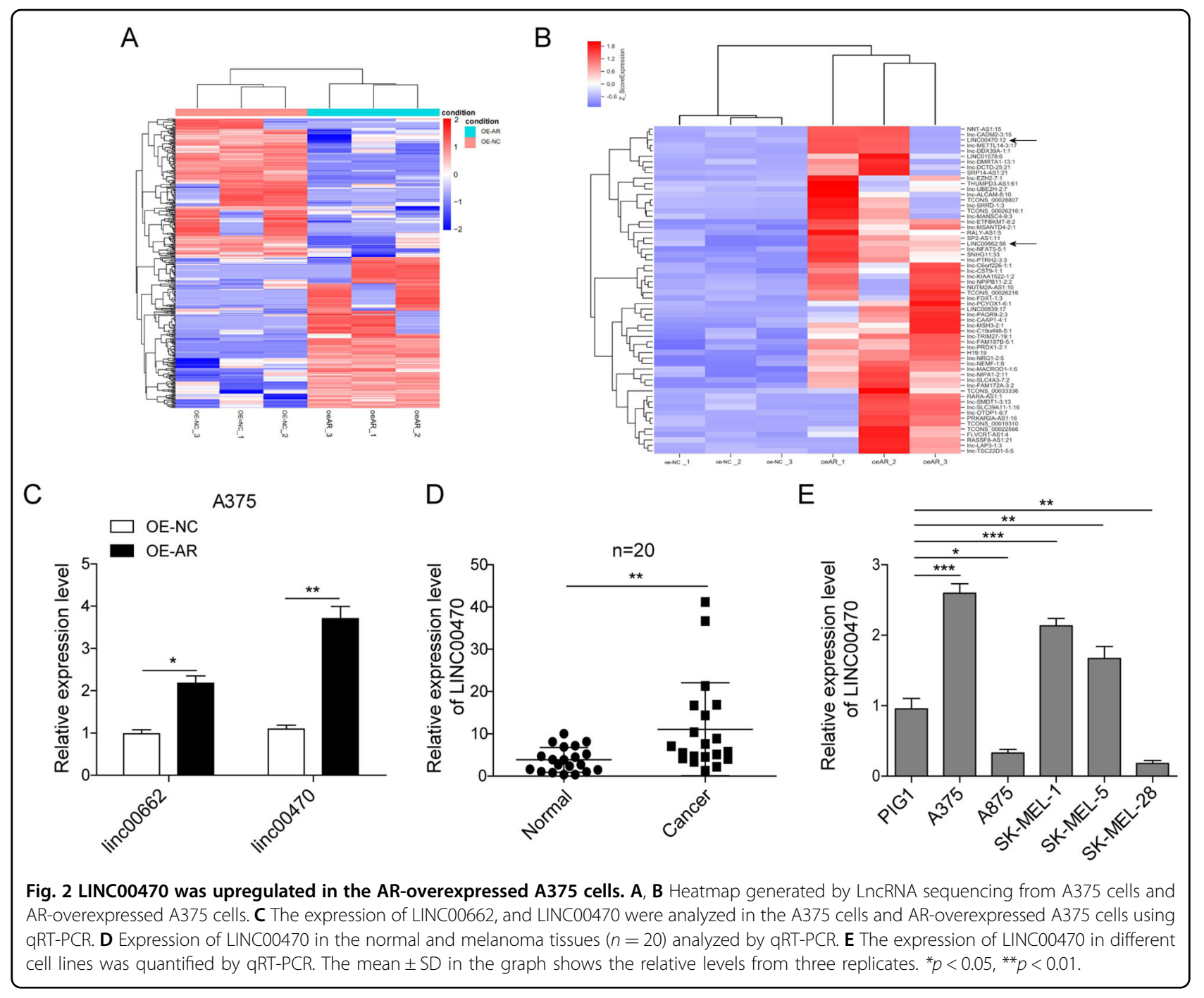

OE of APEX1 reversed the effect of silencing LINC00470 in cells (Fig. 4C-F). Furthermore, we found that knockdown LINC00470 inhibited the cell migration ability and OE of APEX1 abrogated the impact of silencing LINC00470 in A375 and SK-MEL-5 cells (Fig. 4G, H). All these results demonstrated that LINC00470 promoted cell proliferation and migration partly by enhancing the protein expression of APEX1 in melanoma cells.

\section{LINC00470 regulated APEX1 expression through activating Zinc finger protein 131 (ZNF131)}

To explore the interaction mechanism between LINC00470 and APEX1, we found that the transcription factor binding with LINC00470 might be ZNF131 by bioinformatic analysis. Then, the relationship among LINC00470, APEX1, and ZNF131 in melanoma cells was detailly investigated by RIP assay and dual-luciferase assay. As shown in Fig. 5A, the result verified that LINC00470 were more enriched in the melanoma cancer tissues relative to the normal tissues when enriched by ZNF131 antibody (Fig. 5A). Meanwhile, the enrichment of LINC00470 and ZNF131 was increased when the A375 cells were overexpressed LINC00470 and ZNF131, or AR (Fig. 5B, C). Furthermore, RNA pull-down assay showed that the stronger binding interaction between LINC00470 and ZNF131 was observed when the LINC00470 was upregulated (Fig. 5D). In order to investigate the interaction between ZNF131 and APEX1, the CHIP assay was performed. The result in Fig. 5E demonstrated that there was a strong interaction between ZNF131 and APEX1 when LINC00470 and ZNF131 were overexpressed in A375 cells. Furthermore, a dual luciferase assay showed that ZNF131 OE significantly decreased the luciferase activity of the APEX promotor but not the APEX promotor-MUT (Fig. 5F). In addition, $\mathrm{OE}$ ZNF131 suppressed the mRNA expression of APEX1 but promoted the protein levels of APEX1 (Fig. 5G, H). As shown in Fig. 5I, J, the results show that ZNF131 was 


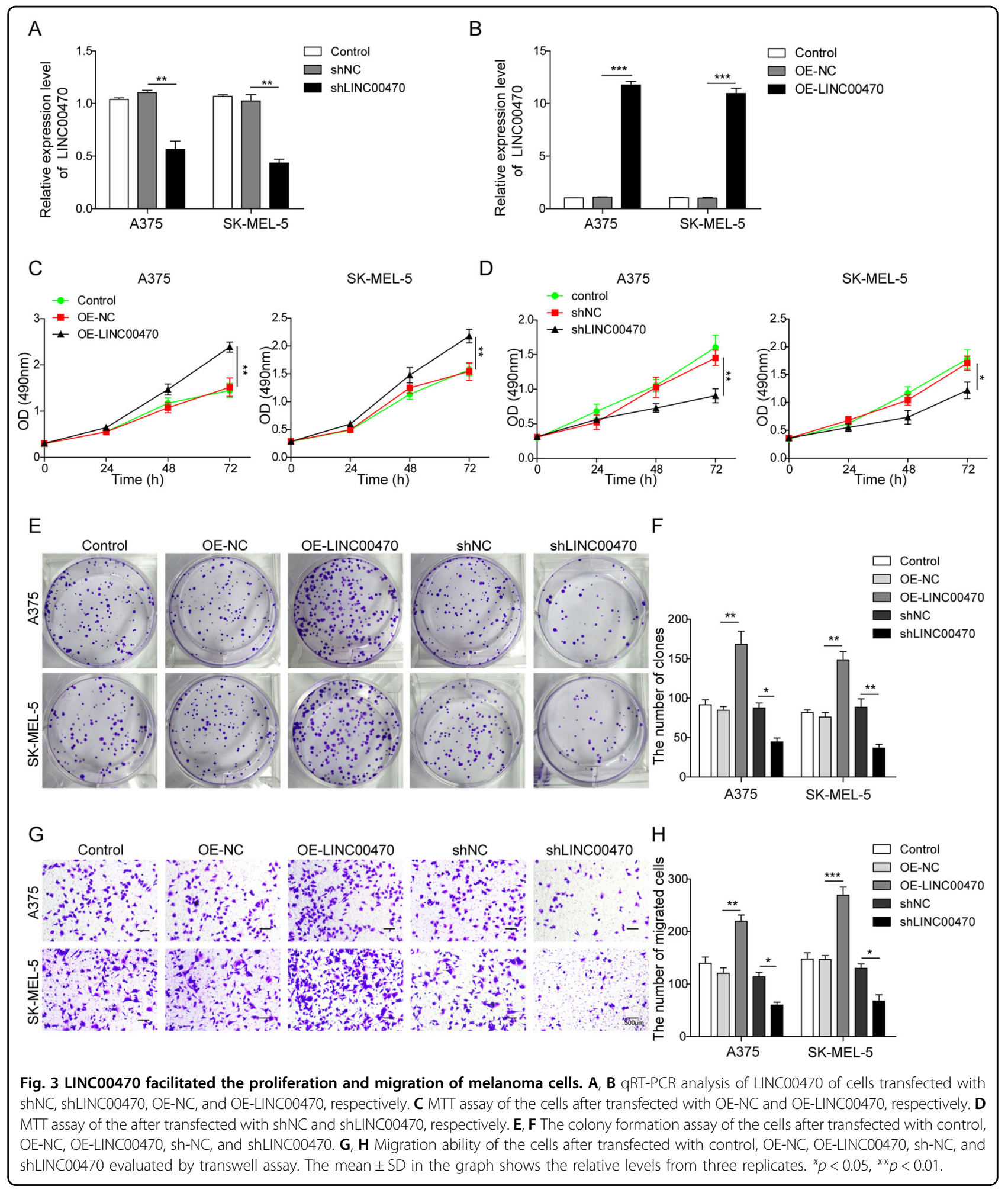

upregulated while APEX1 was downregulated in melanoma tissues compared with the normal tissue. However, the western blot and IHC analysis demonstrated that both APEX1 and ZNF131 proteins were significantly overexpressed in the melanoma tissues (Fig. 5K-M). Specifically, we noticed that the protein expression of APEX1 was not consistent with mRNA expression level in melanoma cells as same as melanoma tissue. Therefore, 


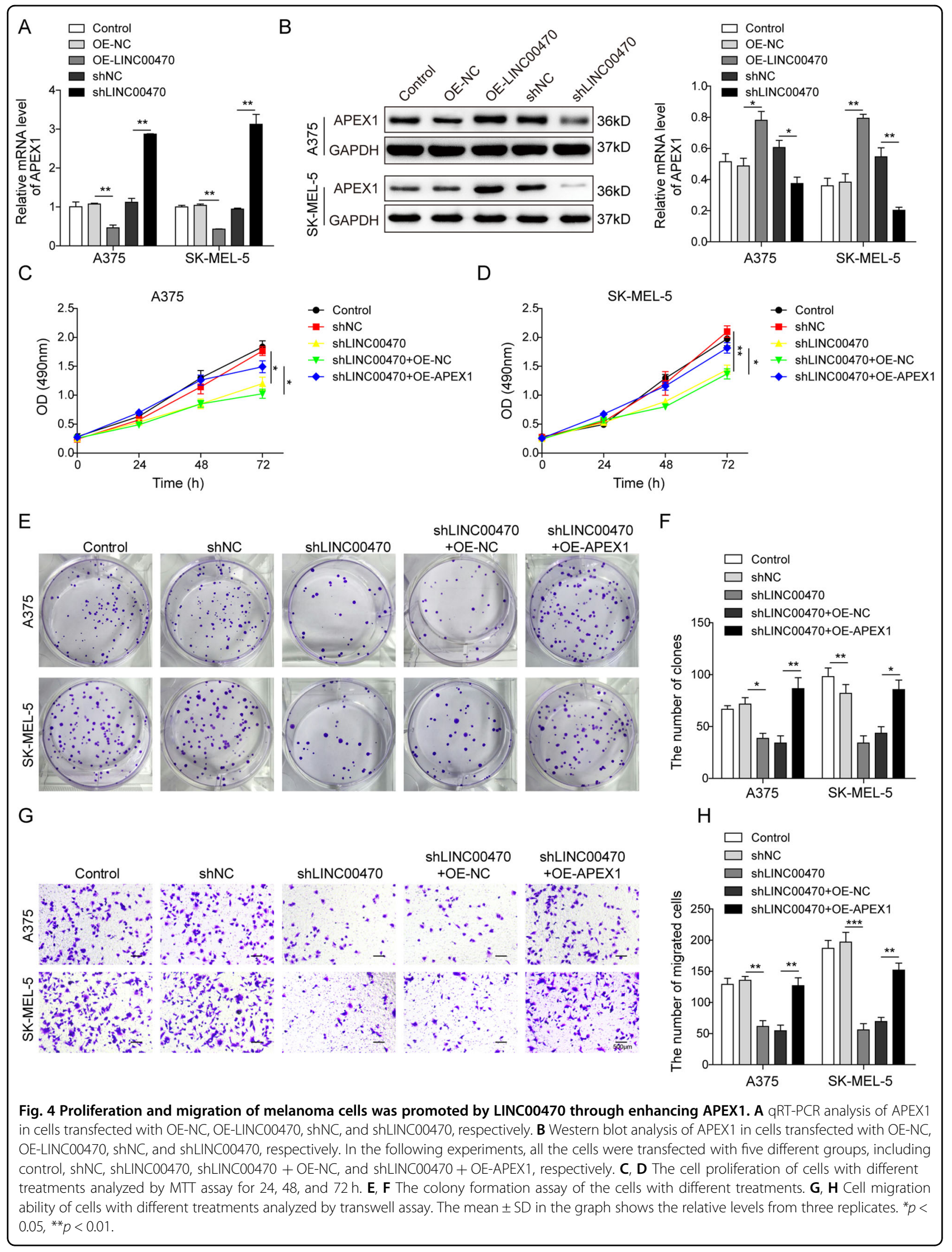




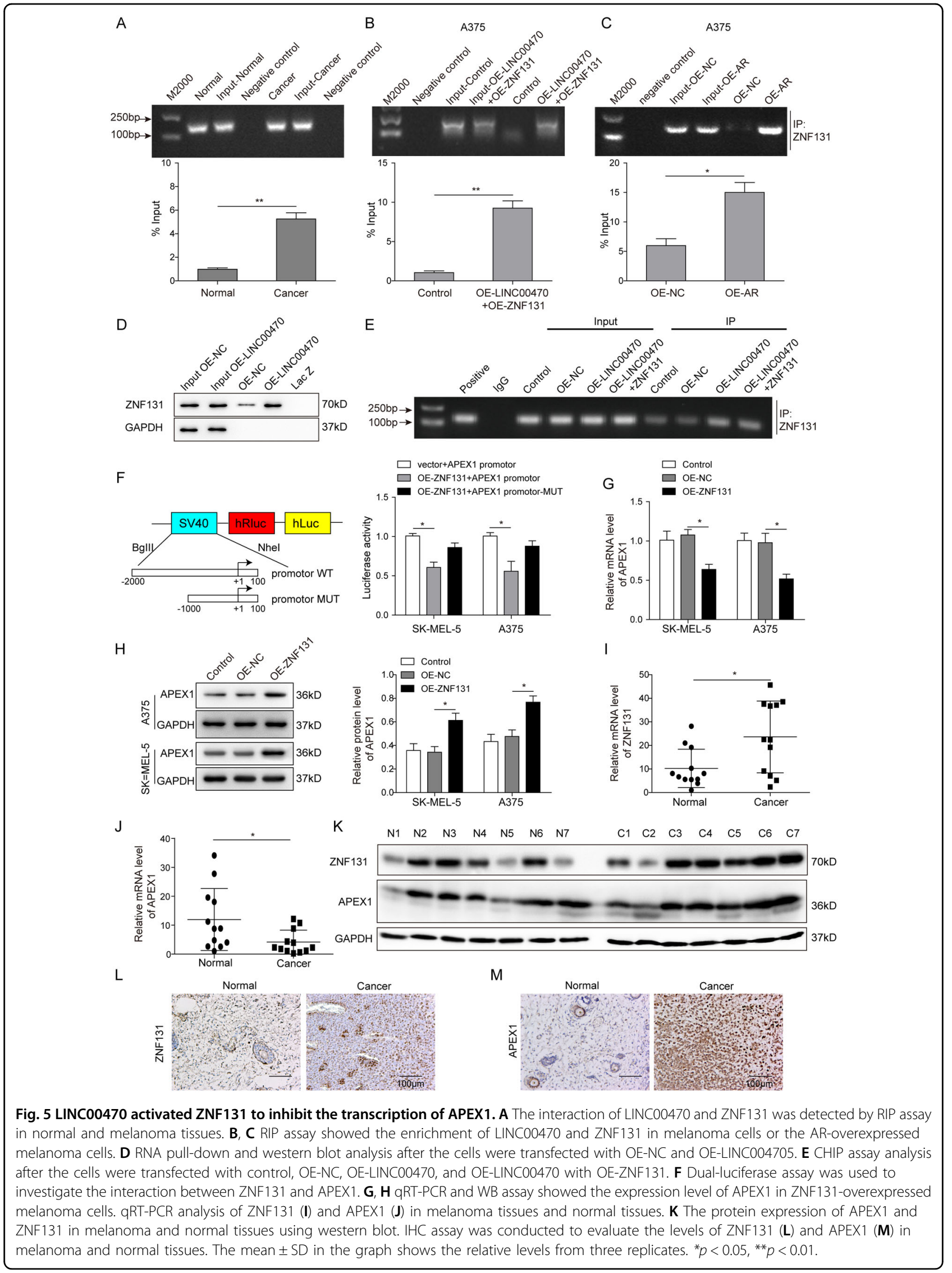




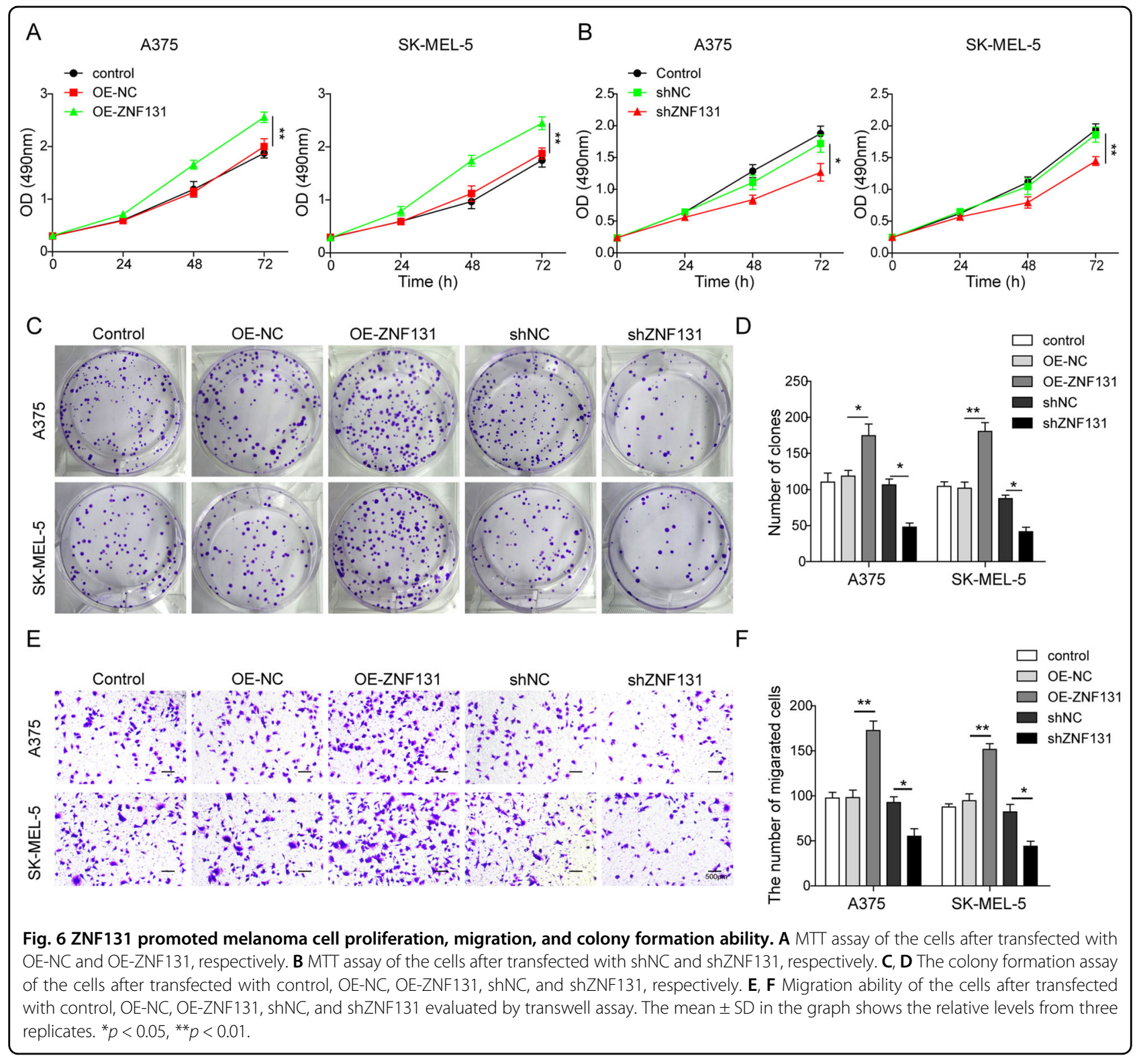

we concluded that LINC00470 could activate the transcription factor ZNF131 to suppress the transcription level of APEX1 but promote the expression of APEX1 at the protein level.

\section{ZNF131 promoted the proliferation and migration of melanoma cells}

To investigate the function of ZNF131 in melanoma, cell proliferation, migration, and colony formation ability were evaluated after the OE or silence of ZNF131 in A375 and SK-MEL-5 cells. As shown in Fig. 6A-D, the OE of ZNF131 significantly promoted the proliferation of melanoma cells. In contrast, cell proliferation was inhibited when ZNF131 was silenced. Furthermore, ZNF131 overexpression improved the cell migration ability while silencing ZNF131 significantly suppressed cell migration (Fig. 6E, F). All these results suggested that the transcription factor ZNF131 could promote melanoma cell proliferation and migration ability.

\section{LINC00470 promoted melanoma proliferation by enhancing APEX1 expression in vivo}

All the above results were used to verify the function of LINC00470 in vitro, to investigate the important role of LINC00470 in vivo, A375 cells transfected with shNC, shLINC00470, shLINC00470 with OE-NC, and shLINC00470 with OE-APEX1, were injected into male nude mice subcutaneously to generate the xenograft model in vivo. As shown in Fig. 7A-C, silencing LINC00470 significantly suppressed the growth of the 


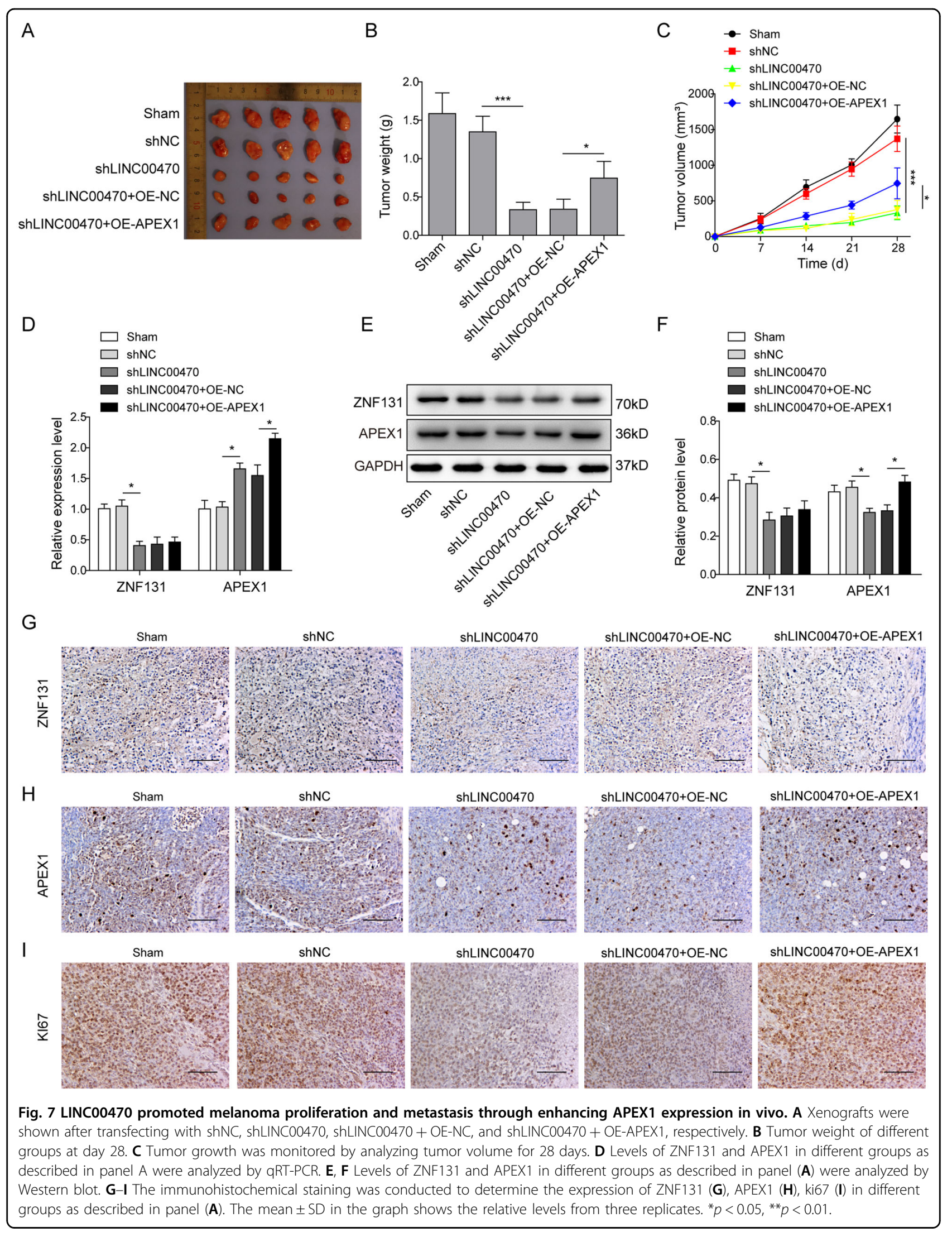


tumor. However, the OE of APEX1 could partly reverse the impact of the silencing LINC00470 (Fig. 7A-C). In addition, we found that knockdown of LINC00470 significantly decreased the mRNA expression of ZNF131 but increased the mRNA expression level of APEX1 (Fig. 7D). However, silencing LINC00470 significantly suppressed the protein expression of ZNF131 and APEX1. Meanwhile, OE of APEX1 significantly enhanced the protein expression of APEX1 but not ZNF131 (Fig. 7E-H). We also found that silencing of LINC00470 significantly suppressed the expression of ki67, which was reversed by OE of APEX1 (Fig. 7I). Overall, these results demonstrated that LINC00470 could promote melanoma tumor growth through regulating APEX1.

\section{Discussion}

In the last decade, LncRNAs have been identified as important regulators in human cancers with different functions ${ }^{29}$. Due to their abnormal expression in tumor tissues and cells, LncRNAs are recognized as a group of promising biomarkers for the diagnosis and prognosis of a variety of cancers ${ }^{22}$. For instance, LncHOTAIR is upregulated in melanoma and is correlated with cancer metastasis and poor prognosis. Knockdown of LncHOTAIR significantly inhibited motility and metastasis ${ }^{30}$. In melanoma tissues and cells, Tian et al. ${ }^{31}$ found that LncRNA MALAT1 was highly expressed. Meanwhile, they demonstrated that the migration ability of melanoma cells was suppressed by silencing LncRNA MALAT1 in vitro. In the current study, we identified that LINC00470 was significantly upregulated in melanoma cells and tissues compared with the normal cells and tissues. The highly expressed LINC00470 was associated with the proliferation and migration ability of advanced melanoma cells, resulting in a rapid tumor growth rate in vivo. Knockdown of LINC00470 significantly suppressed the melanoma cell proliferation and migration, as well as the tumor growth in vivo. Therefore, our results concluded that LINC00470 served as an oncogenic LncRNA in melanoma.

Previously, LINC00470 was found to regulate glioblastoma development through mediating cell autophagy $^{26,27}$. In another report, LINC00470 promoted the HCC proliferation through interaction with NF45/NF90 complex, which regulates gene expression and mRNA stability $^{25}$. In gastric cancer, LINC00470 was also highly expressed and facilitated the malignant behavior of gastric cancer cells through degrading PTEN mRNA ${ }^{28}$. The mechanism of LINC00470 in regulating the proliferation and migration in melanoma is still unknown. Herein, we found that LINC00470 promoted the proliferation and migration of melanoma cells by facilitating the expression of APEX1. APEX1 is a protein that has two functions, including the regulation of DNA repair under oxidative stress and protein reduction-oxidation function ${ }^{32}$. Recently, APEX1 is recognized as a potential diagnostic biomarker in various cancers, such as renal carcinoma, hepatobiliary carcinomas, cholangiocarcinoma, non-small-cell lung cancer, etc. ${ }^{8-10,32}$. For example, Wang et al. ${ }^{10}$ showed that the expression of APEX1 was significantly higher in NSCLC tumor tissues than that in the normal tissues, which could be regulated by the miR-296$3 p$ in NSCLC. In the present study, we revealed that LINC00470 was regulating APEX1 to promote the proliferation and migration of melanoma cells, resulting in significant tumor growth.

However, the regulating mechanism of LINC00470 for APEX1 was still unclear in melanoma. Herein, through bioinformatic analysis, RIP assay, and dualluciferase assay, we identified that LINC00470 activated the transcription factor ZNF131 to regulate APEX1. ZNF131, a member of the zinc finger protein superfamily, is found to act as a transcriptional regulator ${ }^{33}$. In cultured cells, ZNF131 was localized in nuclear, which was found to mediate transcription repression ${ }^{34}$. For instance, Han et al..$^{35}$ reported that ZNF131 inhibited estrogen receptor $\alpha(E R \alpha)$-mediated transcriptional activity in a dose-dependent manner. Chung et al. demonstrated that ZNF131 bonded with estrogen receptors to suppress estrogen signaling, which further inhibited breast cancer cell proliferation ${ }^{36}$. Ding et al. ${ }^{37}$ discovered that ZNF131 suppressed centrosome fragmentation in glioblastoma by targeting HAUS5. In melanoma cells, we found that ZNF131 targeted APEX1, which finally promoted melanoma cell proliferation and migration. Interestingly, we noticed that the ZNF131 inhibited the mRNA level of APEX1. In contrast, the protein level of APEX1 was upregulated in melanoma tissues, indicating that other regulatory mechanisms to the target gene may exist except the LINC00470/ZNF131/APEX1 axis. The detailed mechanism needs further investigation in the future.

LncRNAs involve in biological processes by a variety of mechanisms, such as chromatin remodeling, cell cycle control, mRNA decay, splicing regulation, and translational regulation ${ }^{38,39}$. However, it is the first time to discover that LINC00470 regulates the target gene through activating the transcription factor, to the best of our knowledge. Therefore, we believe that our present study promotes the understanding of the mechanism of LncRNA in regulating the pathogenesis of melanoma.

In summary, our study demonstrated that LINC00470 could serve as a promising potential therapeutic target for melanoma patients. The detailed investigation about the molecular mechanisms indicated that LINC00470 promoted the proliferation and migration of melanoma cells by facilitating the APEX1 via regulating ZNF131. 


\section{Acknowledgements}

We would like to give our sincere gratitude to the reviewers for their constructive comments. This work was supported by Hunan Provincial Key Research and Development Plan (No. 2016SK2092).

\section{Author contributions}

J.-H.L.: guarantor of integrity of the entire study. J.-H.L. and T.H.: study concepts. Y.-J.W. and T.H.: study design. T.H.: definition of intellectual content. T.H.: literature research. T.H. and M.-T.H.: experimental studies. T.H., X.-J.L., and W.-Y. T.: data acquisition. T.H.: data analysis. T.H.: statistical analysis. T.H.: paper preparation. Y.-J.W.: paper editing. J.-H.L.: a paper review.

\section{Ethics statement}

Approvements were made by the ethics committee of Central South University. All patients were informed of the study and signed the written consent. The animal experiments were approved by the Animal Ethics Committee of Central South University.

\section{Conflict of interest}

The authors declare that they have no conflict of interest.

\section{Publisher's note}

Springer Nature remains neutral with regard to jurisdictional claims in published maps and institutional affiliations.

Supplementary information The online version contains supplementary material available at https://doi.org/10.1038/s41419-021-03612-z.

Received: 12 November 2020 Revised: 5 March 2021 Accepted: 10 March 2021

Published online: 19 April 2021

\section{References}

1. MacKie, R. M., Hauschild, A. \& Eggermont, A. M. Epidemiology of invasive cutaneous melanoma. Ann. Oncol. 20, vi1-vi7 (2009).

2. Richtig, E. et al. Clinical epidemiology of invasive cutaneous malignant melanoma in the Austrian province Styria in the years 2001-2003 and its relationship with local geographical, meteorological and economic data. Dermatology 214, 246-252 (2007).

3. Xiao, W. \& Yin, A. LINC0638 IncRNA is involved in the local recurrence of melanoma following surgical resection. Oncol. Lett. 18, 101-108 (2019).

4. Trotter, S. C., Sroa, N., Winkelmann, R. R., Olencki, T. \& Bechtel, M. A global review of melanoma follow-up guidelines. J. Clin. Aesthet. Dermatol. 6, 18-26 (2013).

5. Pacheco, I., Buzea, C. \& Tron, V. Towards new therapeutic approaches for malignant melanoma. Expert Rev. Mol. Med. 13, e33 (2011).

6. Zhang, H. et al. LncRNA HOXD-AS1 promotes melanoma cell proliferation and invasion by suppressing RUNX3 expression. Am. J. Cancer Res. 7, 2526-2535 (2017).

7. Tell, G., Damante, G., Caldwell, D. \& Kelley, M. R. The intracellular localization of APE1/Ref-1: more than a passive phenomenon? Antioxid. Redox Signal. 7, 367-384 (2005).

8. Kim J. M. et al. APEX1 expression as a potential diagnostic biomarker of clear cell renal cell carcinoma and hepatobiliary carcinomas. J. Clin. Med. 8, 2019.

9. Cao, L., Cheng, H., Jiang, Q., Li, H. \& Wu, Z. APEX1 is a novel diagnostic and prognostic biomarker for hepatocellular carcinoma. Aging 12, 4573-4591 (2020).

10. Wang, L., Chen, R. \& Zhang, Y. miR-296-3p targets APEX1 to suppress cell migration and invasion of non-small-cell lung cancer. Oncol. Lett. 18, 2612-2618 (2019).

11. Woo, J. et al. Prognostic value of human apurinic/apyrimidinic endonuclease 1 (APE1) expression in breast cancer. PLoS ONE 9, e99528 (2014).

12. Kim, H. B. et al. Clinical significance of jagged-1 activated by APEX1 as a chemoresistance factor in advanced gastric cancer. Anticancer Res. 40, 1897-1904 (2020).

13. Londero, A. P. et al. Expression and prognostic significance of APE1/Ref-1 and NPM1 proteins in high-grade ovarian serous cancer. Am. J. Clin. Pathol. 141, 404-414 (2014).
14. Fishel, M. L. et al. Antitumor activity and mechanistic characterization of APE1/ Ref-1 inhibitors in bladder cancer. Mol. Cancer Ther. 18, 1947-1960 (2019).

15. Kim, M. H. et al. Colon cancer progression is driven by APEX1-mediated upregulation of Jagged. J. Clin. Investig. 8, 3211-3230 (2013).

16. Kelley, M. R. et al. Elevated and altered expression of the multifunctional DNA base excision repair and redox enzyme Ape1/ref-1 in prostate cancer. Clin. Cancer Res. 7, 824-830 (2001).

17. Wang, D., Luo, M. \& Kelley, M. R. Human apurinic endonuclease 1 (APE1) expression and prognostic significance in osteosarcoma: enhanced sensitivity of osteosarcoma to DNA damaging agents using silencing RNA APE1 expression inhibition. Mol. Cancer Ther. 3, 679-686 (2004).

18. Yang, S., Irani, K., Heffron, S. E., Jurnak, F. \& Meyskens, F. L. Jr. Alterations in the expression of the apurinic/apyrimidinic endonuclease-1/redox factor-1 (APE/Ref-1) in human melanoma and identification of the therapeutic potential of resveratrol as an APE/Ref-1 inhibitor. Mol. Cancer Ther. 4, 1923-1935 (2005).

19. Figl, A. et al. Single nucleotide polymorphisms in DNA repair genes XRCC1 and APEX1 in progression and survival of primary cutaneous melanoma patients. Mutat. Res. 661, 78-84 (2009).

20. Wang, J. et al. LncRNA HOXA-AS2 and its molecular mechanisms in human cancer. Clin. Chim. Acta 485, 229-233 (2018).

21. Botti, $G$. et al. LncRNA HOTAIR as prognostic circulating marker and potential therapeutic target in patients with tumor diseases. Curr. Drug Targets 18, 27-34 (2017).

22. Yu, X., Zheng, H., Tse, G., Chan, M. T. \& Wu, W. K. Long non-coding RNAs in melanoma. Cell Prolif. 51, e12457 (2018).

23. Chen, L. et al. LnCRNA GAS5 is a critical regulator of metastasis phenotype of melanoma cells and inhibits tumor growth in vivo. Onco Targets Ther. 9, 4075-4087 (2016).

24. Stohr, $\mathrm{H}$. et al. EST mining of the UniGene dataset to identify retina-specific genes. Cytogenet. Cell Genet. 91, 267-277 (2000).

25. Huang, W. et al. LnCRNA LINC00470 promotes proliferation through association with NF45/NF90 complex in hepatocellular carcinoma. Hum. Cell 33 131-139 (2020).

26. Liu, C. et al. A cytoplasmic long noncoding RNA LINC00470 as a new AKT activator to mediate glioblastoma cell autophagy. J. Hematol. Oncol. 11, 77 (2018).

27. Liu, C. et al. LINC00470 coordinates the epigenetic regulation of ELFN2 to distract GBM cell autophagy. Mol. Ther. 26, 2267-2281 (2018).

28. Yan, J. et al. LnCRNA LINC00470 promotes the degradation of PTEN mRNA to facilitate malignant behavior in gastric cancer cells. Biochem. Biophys. Res. Commun. 521, 887-893 (2020)

29. Liu, Y. R. et al. Comprehensive transcriptome profiling reveals multigene signatures in triple-negative breast cancer. Clin. Cancer Res. 22, 1653-1662 (2016).

30. Tang, L., Zhang, W., Su, B. \& Yu, B. Long noncoding RNA HOTAIR is associated with motility, invasion, and metastatic potential of metastatic melanoma. Biomed. Res. Int. 2013, 251098 (2013).

31. Tian, Y., Zhang, X., Hao, Y., Fang, Z. \& He, Y. Potential roles of abnormally expressed long noncoding RNA UCA1 and Malat-1 in metastasis of melanoma. Melanoma Res. 24, 335-341 (2014).

32. Tummanatsakun, D. et al. Serum apurinic/apyrimidinic endodeoxyribonuclease 1 (APEX1) level as a potential biomarker of cholangiocarcinoma. Biomolecules 9, 413 (2019).

33. Donaldson, N. S. et al. Kaiso regulates Znf131-mediated transcriptional activation. Exp. Cell Res. 316, 1692-1705 (2010).

34. Donaldson, N. S., Daniel, Y., Kelly, K. F., Graham, M. \& Daniel, J. M. Nuclear trafficking of the POZ-ZF protein Znf131. Biochim. Biophys. Acta 1773, 546-555 (2007).

35. Han, X. et al. High-throughput cell-based screening reveals a role for ZNF131 as a repressor of ERalpha signaling. BMC Genomics 9, 476 (2008).

36. Oh, Y. \& Chung, K. C. Zinc finger protein 131 inhibits estrogen signaling by suppressing estrogen receptor alpha homo-dimerization. Biochem. Biophys. Res. Commun. 430, 400-405 (2013).

37. Ding, Y. et al. ZNF131 suppresses centrosome fragmentation in glioblastoma stem-like cells through regulation of HAUS5. Oncotarget 8, 48545-48562 (2017).

38. Xia, T., Xiao, B. X. \& Guo, J. M. Acting mechanisms and research methods of long noncoding RNAs. Yi Chuan 35, 269-280 (2013).

39. Wapinski, O. \& Chang, H. Y. Long noncoding RNAs and human disease. Trends Cell Biol. 21, 354-361 (2011). 